\title{
An Ironman triathlon reduces neuromuscular performance due to impaired force transmission and reduced leg stiffness
}

\author{
Mueller, Sandro Manuel ; Knechtle, Patrizia ; Knechtle, Beat ; Toigo, Marco
}

DOI: https://doi.org/10.1007/s00421-014-3051-2

Posted at the Zurich Open Repository and Archive, University of Zurich ZORA URL: https://doi.org/10.5167/uzh-102133

Journal Article

Published Version

Originally published at:

Mueller, Sandro Manuel; Knechtle, Patrizia; Knechtle, Beat; Toigo, Marco (2015). An Ironman triathlon reduces neuromuscular performance due to impaired force transmission and reduced leg stiffness. European Journal of Applied Physiology, 115(4):795-802.

DOI: https://doi.org/10.1007/s00421-014-3051-2 


\title{
An Ironman triathlon reduces neuromuscular performance due to impaired force transmission and reduced leg stiffness
}

\author{
Sandro Manuel Mueller • Patrizia Knechtle • \\ Beat Knechtle $\cdot$ Marco Toigo
}

Received: 18 August 2014 / Accepted: 10 November 2014 / Published online: 4 December 2014

(C) Springer-Verlag Berlin Heidelberg 2014

\begin{abstract}
Purpose An Ironman triathlon is associated with changes in body composition as well as decreases in neuromuscular function. While the changes in body composition occurring during an Ironman are well investigated, comprehensive data on the changes in neuromuscular performance are scarce. In the present study, we investigated the mechanical alterations underlying reported reductions in maximal muscular force and power after an Ironman race in men.

Methods Before and directly after an Ironman, countermovement jump (CMJ), squat jump (SJ), and multiple one-legged hopping (m1LH) maneuvers were performed to assess fatigue-related alterations in mechanical variables in thirteen male non-professional triathletes.

Results During CMJ, peak power $(P=0.003)$, peak velocity $(P<0.001)$, jump height $(P=0.007)$, and rate of force development $(P=0.042)$ decreased during the Ironman. Total $(P<0.001)$ and positive $(P=0.003)$ impulses during a $\mathrm{CMJ}$ were reduced after the triathlon, while both negative impulses did not differ pre to post
\end{abstract}

Communicated by Peter Krustrup.

S. M. Mueller · M. Toigo $(\square)$

Exercise Physiology Lab, Institute of Human Movement

Sciences, ETH Zurich, Winterthurerstrasse 190, 8057 Zurich,

Switzerland

e-mail: marco.toigo@ hest.ethz.ch

P. Knechtle $\cdot$ B. Knechtle

Institute of General Practice and Health Services Research,

University of Zurich, Zurich, Switzerland

M. Toigo

Department of Orthopaedics, Laboratory for Muscle Plasticity, University of Zurich, Balgrist University Hospital, Zurich,

Switzerland
Ironman. Absolute peak force remained constant during CMJ $(P=0.200)$ and SJ $(P=0.764)$. Maximal voluntary ground reaction force $\left(F_{\mathrm{m} 1 \mathrm{LH},} P<0.001\right)$ and peak stiffness $(P=0.003)$ during $\mathrm{m} 1 \mathrm{LH}$ were decreased after the Ironman.

Conclusions The reduced CMJ height was a result of the lower positive impulse. Therefore, the neuromuscular deficit after the Ironman race was due to impairments in force transmission, resulting in a lower average positive force during CMJ, because of a slower rate of force development. The decreased $F_{\text {m1LH }}$ could be partly explained by reduced leg stiffness.

Keywords Countermovement jump - Multiple onelegged hopping $\cdot$ Impulse $\cdot$ Rate of force development
Abbreviations
CMJ Countermovement jump
$F_{\text {m1LH }}$ Maximal voluntary ground reaction force
$I_{1} \quad$ Negative impulse 1
$I_{2} \quad$ Positive impulse 2
$I_{3} \quad$ Negative impulse 3
m1LH Multiple one-legged hopping
SJ Squat jump

\section{Introduction}

Long-distance triathlons are some of the most strenuous and fatiguing sport events. The most known and most popular long-distance triathlon event-the Ironman-consists of $3.8 \mathrm{~km}$ swimming, $180 \mathrm{~km}$ cycling, and $42.2 \mathrm{~km}$ running. During competition time, which exceeds $7.5 \mathrm{~h}$ of continuous exercise, the body is stressed severely. This exercise-induced stress is associated with 
changes in body composition as well as alterations in neuromuscular performance. Body composition changes during an Ironman are well investigated. These changes comprise fluid loss (Hiller et al. 1987), glycogen depletion (Gillum et al. 2006; Knechtle et al. 2010) and decreases in fat mass (Mueller et al. 2013). Contrary to these investigations, comprehensive data on the changes in neuromuscular performance are scarce. Up to date, it was shown that a long-distance triathlon event led to a reduced capacity of force generation during knee extension and flexion in competitive male triathletes (Margaritis et al. 1999). Furthermore, it was shown that 1 day after an Ironman maximal isometric force during knee extension as well as squat jump (SJ) and countermovement jump (CMJ) height were reduced in well-trained male triathletes (Suzuki et al. 2006). The decrease in CMJ height, together with a decrease in mean jump power was also found to occur after a half-Ironman triathlon in well-trained triathletes (Coso et al. 2012).

The underlying mechanisms leading to the decreases in peak force and jump height after an Ironman remain inscrutable. To our knowledge, only one study addressed this issue so far showing that contraction and relaxation times, as measured by tensiomyography, were prolonged and muscle stiffness was reduced after an Ironman in experienced ultra-endurance athletes (Garcia-Manso et al. 2011). Interestingly, different results were obtained for muscles with different function. While pronounced fatigue occurred in the $M$. biceps femoris, hardly any changes in fatigue indices were present in $M$. rectus femoris. This result is rather unexpected, since it is assumed that $M$. quadriceps femoris is the main contributor for the motive force during cycling (Raasch et al. 1997) and running (Montgomery et al. 1994).

Knowledge of the factors underpinning neuromuscular fatigue during an Ironman is an important prerequisite to design and plan training routines with the goal to postpone and minimize fatigue. In this study, we investigated the mechanical alterations underlying the reduction in maximal muscular force and power after an Ironman race in men. In addition, we investigated if maximal voluntary ground reaction force during one-legged hopping (1LH), which serves as a measure for maximal muscle force, is also reduced after an Ironman. We hypothesized that maximal voluntary ground reaction force would be reduced because of a lower stiffness of the legs. In comparison to $1 \mathrm{LH}$, two-legged jumping yields only submaximal values for force. Thus, we hypothesized that peak force during a two-legged CMJ would not be decreased after an Ironman and that the expected reduction in jump height and power would be solely due to a lower peak velocity.

\section{Methods}

Participants

Thirteen male non-professional Ironman triathletes participating in the 'Ironman Switzerland', which was held in Zurich, were recruited for this study. The physical characteristics of the participants were (mean $\pm \mathrm{SD}$ ): age $40.1 \pm 6.4$ years, body mass $72.7 \pm 8.3 \mathrm{~kg}$, and height $175.9 \pm 4.4 \mathrm{~cm}$. The participants had on average $4.5 \pm 4.1$ years experience with Ironman races. The participants were fully informed about all applied procedures and about the associated risks. Before participating, all participants completed a routine health questionnaire and signed an informed consent. All experiments were approved by the ethics committee of the canton of Zurich (Switzerland), and the study was performed in accordance with the ethical standards laid down in the Declaration of Helsinki for human experimentation. Eight of these athletes also participated in our study, in which we investigated the changes in body composition after an Ironman race (Mueller et al. 2013).

Experimental procedure and race day

The participants reported to the laboratory $2-3$ days before the competition for the determination of baseline characteristics. Directly after the race $(\sim 3 \mathrm{~h})$, participants reported to the laboratory for the second visit. During both visits, the participants conducted three two-legged CMJ, three two-legged SJ, and two multiple 1LH (m1LH) of the dominant leg. Water temperature was $20^{\circ} \mathrm{C}$. At the beginning of the cycling stage, air temperature was $14{ }^{\circ} \mathrm{C}$ and humidity was $69 \%$. During the afternoon, air temperature raised to $23{ }^{\circ} \mathrm{C}$ and humidity decreased to $37 \%$. The participants completed the Ironman in 11:08:20 $\pm 1: 22: 47 \mathrm{~h}$ :min:s. The split times for the swimming, cycling, and running stages were 1:14:30 \pm 0:09:36, 5:36:21 $\pm 0: 35: 46$, and 4:10:12 \pm 0:43:04 h:min:s, respectively (transition times not included).

\section{Jumping mechanography}

Three vertical CMJ with freely moving arms (separated by $30 \mathrm{~s}$ of rest) were performed on a strain gauge ground reaction force platform (Leonardo Mechanograph ${ }^{\circledR}$, Novotec, Pforzheim, Germany) linked to a desktop computer using an integrated analog digital board and software system (Leonardo Mechanography GRFP version 4.2, Novotec, Pforzheim, Germany). The participants were instructed to remove their shoes, stand with feet shoulder width apart, and arms hanging loosely at their sides. They were further 
instructed to jump as high as possible while keeping their head still during the flight, and to stand still again with arms hanging loosely after landing. After the CMJs and 1 min rest, participants performed three vertical SJ with the hands held in place on the hips (separated by $30 \mathrm{~s}$ of rest). Both, CMJ and SJ, were performed, because the two jumping maneuvers assess different types of mechanical power, which in turn reflect the different types of power used in a triathlon race. CMJ assesses 'reactive' power (i.e., force and velocity are phase-shifted by $90^{\circ}$ ), while $\mathrm{SJ}$ assesses 'real' power (i.e., force and velocity are in-phase). Cycling power is real power (net power $>0$ ), while running is characterized by reactive power (net power $=0$ ). From the three valid attempts each (CMJ and SJ), the jump with the highest jump height was used for further analyses. Prior to the two-legged jumping tests, body mass was calculated from ground reaction force measurements while still standing on the force plate for $2 \mathrm{~s}$. Finally, maximum voluntary ground reaction force $\left(F_{\mathrm{m} 1 \mathrm{LH}}\right)$ was determined by m1LH (dominant leg) as previously described (Anliker et al. 2011; Anliker and Toigo 2012). In brief, the participants jumped repeatedly ( $\sim 15$ jumps) on the forefoot with a stiff knee and without touching the ground with their heels. Any jumps with heel contact were excluded from the analysis. Heel contact was controlled visually during the jumping maneuver. $F_{\mathrm{m} 1 \mathrm{LH}}$ corresponded to peak force during $\mathrm{m} 1 \mathrm{LH}$. The typical error, expressed as a coefficient of variation, determined in our laboratory for $F_{\mathrm{mlLH}}$ and two-legged peak jump power is 5.3 and $3.7 \%$, respectively.

\section{Data analysis}

For both CMJ and SJ, peak jump height was calculated as the highest displacement of the center of mass, and peak power was calculated from the product of force and velocity. Minimal height was calculated as the highest negative displacement of the center of mass during the jumping maneuvers. Rate of force development during CMJ was calculated as $\Delta$ force divided by $\Delta$ time, whereby $\Delta$ force was the difference between the highest and lowest force during the take-off phase of the jumping maneuver and $\Delta$ time represents the time interval between these two occurrences. For the impulse analysis, baseline force was determined while the participants stood still on the ground reaction force plate for $1 \mathrm{~s}$. During a CMJ, the measured ground reaction force intersected three times with this baseline value resulting in three areas under the force curve. The three individual impulses $\left(I_{1}, I_{2}, I_{3}\right)$ were calculated correspondingly as the areas between the progression of the ground reaction force curve and the baseline force (Fig. 1). The total impulse was calculated as the sum of the two negative $\left(I_{1}, I_{3}\right)$ impulses and the positive impulse $\left(I_{2}\right)$, i.e., the sum was calculated as $I_{2}-I_{1}-I_{3}$. We used Matlab

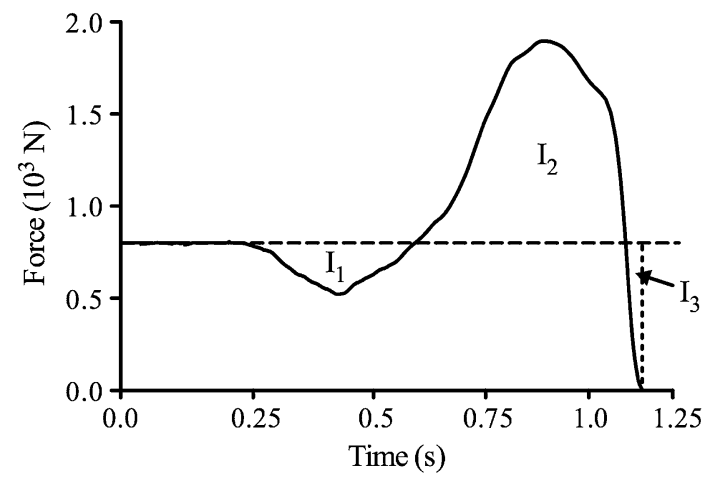

Fig. 1 Progression of ground reaction force during countermovement jumping with the areas between progression curve and baseline (dashed line) representing the three impulses $\left(I_{1}, I_{2}, I_{3}\right)$. Dotted line represents the time point of take-off and end of the 3rd impulse, respectively

R2013a (MathWorks, Natick, MA, USA) for the impulse analysis.

Statistics

Data are presented as mean values \pm SD. Normality of data and homogeneity of variance were visually ascertained by Q-Q plots and Levene's test for equality of variances, respectively. To assess differences in jumping mechanography values, a two-way repeated-measures analysis of variance having one level of condition and two levels of time (pre and post) was used. The assumption of sphericity was tested using Mauchly's test. The null hypothesis for the Mauchly's test is that the error covariance matrix of the dependent variable is proportional to an identity matrix. If the probability of the test statistic is greater than or equal to the level of significance, the null hypothesis is rejected and the assumption is not violated. In the case of violation of the assumption of sphericity, degrees of freedom were corrected using Greenhouse-Geisser corrected estimates of sphericity producing an $\mathrm{F}$ ratio with reduced Type I error rate. We used SPSS 20.0 statistical software (SPSS, Chicago, USA) for all statistical analyses. Statistical significance was set at $P<0.05$.

\section{Results}

Jump height during CMJ was reduced $(-8.8 \pm 9.7 \%$, Fig. 2a) after the race, while it did not differ from pre to post Ironman during a SJ (Table 1). Peak forces during CMJ (Fig. 2b) and SJ were not different pre to post Ironman, respectively (Table 1). Peak velocity decreased during both CMJ (Fig. 2c) and SJ $(-6.1 \pm 4.5$ and $-4.6 \pm 3.1 \%$, respectively), which resulted in a decreased absolute 
Fig. 2 Pre and post Ironman results for countermovement jump a jump height, $\mathbf{b}$ peak force, $\mathbf{c}$ peak velocity, and $\mathbf{d}$ peak power for each participant
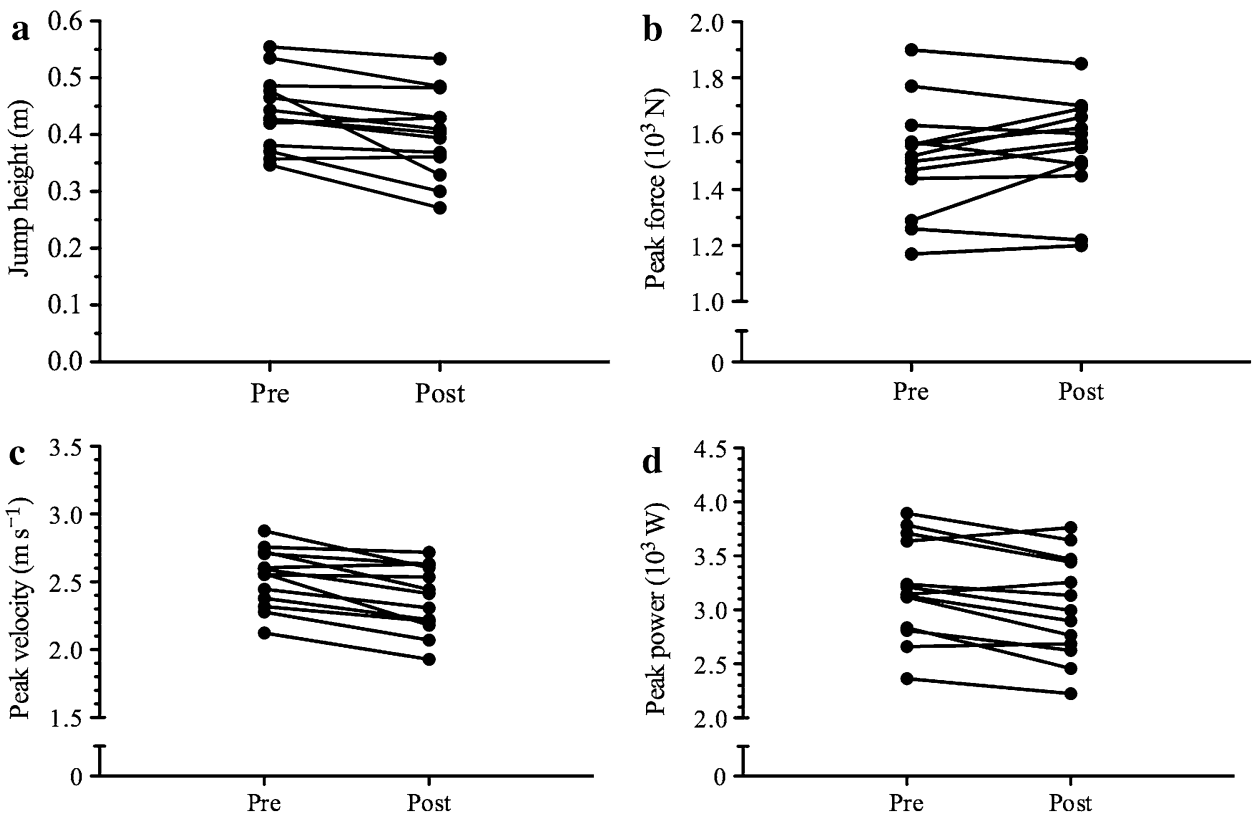

$[-5.2 \pm 5.2$ and $-4.8 \pm 6.1 \%$ for CMJ (Fig. 2d) and SJ, respectively] but not relative peak power $(-2.9 \pm 5.3$ and $-2.6 \pm 6.1 \%$ for CMJ and SJ, respectively) output. Peak velocities correlated with the jump heights pre (Fig. 3a) and post Ironman (Fig. 3b). The positive impulse $I_{2}$ during a CMJ was decreased after the Ironman race (Fig. 4). $I_{1}$ and $I_{3}$ during the CMJ were similar pre to post Ironman $\left(P=0.122\right.$ and $P=0.299$ for $I_{1}$ and $I_{3}$, respectively). The total impulse of the CMJ was decreased significantly (Fig. 4). The CMJ time interval for $I_{2}$ was increased after the Ironman $(0.53 \pm 0.17$ vs. $0.61 \pm 0.21 \mathrm{~s}$ for pre vs. post, $P=0.006$ ). The time intervals for $I_{1}$ and $I_{3}$ were not different from pre to post Ironman $\left(I_{1}: 0.79 \pm 0.27 \mathrm{vs}\right.$. $0.69 \pm 0.36 \mathrm{~s}$ for pre vs. post, $P=0.446 ; I_{3}: 0.03 \pm 0.01$ vs. $0.04 \pm 0.01 \mathrm{~s}$ for pre vs. post, $P=0.136$ ). The rate of force development during CMJ decreased during the Ironman $\left(1.92 \pm 0.71\right.$ vs. $1.70 \pm 0.66 \mathrm{kN} \mathrm{s}^{-1}$ for pre vs. post, $P=0.042)$.

There were no significant differences between relative changes in jump height and peak velocity $(P=0.946), I_{2}$ $(P=0.997)$ and total impulse $(P=0.996$, Fig. 5). After the Ironman, minimal height was reduced during CMJ by $0.06 \pm 0.04 \mathrm{~m}(P<0.001)$ and did not differ from pre to post during $\mathrm{SJ}(0.00 \pm 0.06 \mathrm{~m}, P=0.805)$. The decrease in minimal height during $\mathrm{CMJ}$ correlated with the reduction in peak velocity (Fig. 6). Absolute and relative $F_{\mathrm{m} 1 \mathrm{LH}}$ decreased by $16.8 \pm 7.8$ and $14.8 \pm 7.9 \%$, respectively (Table 1). Peak stiffness during m1LH decreased in response to the Ironman (pre vs. post: $174.3 \pm 54.6$ vs. $\left.133.7 \pm 39.3 \mathrm{~N} \mathrm{~cm}^{-1}, P=0.003\right)$. Body mass decreased during the Ironman by $2.4 \pm 1.2 \%$ (pre vs. post: $72.5 \pm 8.4$ vs. $70.6 \pm 7.9 \mathrm{~kg}, P<0.001)$.

\section{Discussion}

We could show for the first time that an Ironman race decreased $F_{\mathrm{m} 1 \mathrm{LH}}$ but did not impair CMJ and SJ peak forces in men. Relative peak force even increased during $\mathrm{CMJ}$ and was not different pre to post Ironman during SJ. Furthermore, absolute peak power and peak velocity during $\mathrm{CMJ}$ and SJ decreased. As for CMJ, the reduction in peak power and peak velocity was due to the reduction in positive and total impulse, since negative impulses did not differ from pre to post Ironman. During CMJ, rate of force development was reduced leading to a decreased average force. Jumping height was lowered during CMJ but was not affected by the Ironman during SJ.

The decrease in CMJ height after the Ironman could be attributed to a lower total impulse during jumping. This decrease in total impulse was based on a reduction in $I_{2}$, while both $I_{1}$ and $I_{3}$ were not different pre to post Ironman. Theoretically, possible explanations for a lower total impulse are shorter impulse durations, a lower peak force and/or a decreased average force. A shorter time interval of the individual impulses was not an explanation for the lower total impulse in this study because the impulse intervals were not different $\left(I_{1}\right.$ and $\left.I_{3}\right)$ or increased $\left(I_{2}\right)$ from pre to post Ironman. A lower peak force also is no explanation in the present case since peak forces were similar pre and post Ironman. Therefore, the cause for the decrease in total impulse was a lower average force during the jumping maneuver, notably without any change in peak force. This altered force kinetic was due to the reduced peak velocity, which was not only reduced during CMJ but also during SJ. These results are supported by the impaired rate of 
Table 1 Results of countermovement jump (CMJ), squat jump (SJ), and multiple one-legged hopping (m1LH) pre and post Ironman

\begin{tabular}{|c|c|c|c|c|c|c|}
\hline & \multicolumn{2}{|l|}{ CMJ } & \multicolumn{2}{|l|}{ SJ } & \multicolumn{2}{|l|}{ m1LH } \\
\hline & Pre & Post & Pre & Post & Pre & Post \\
\hline \multicolumn{7}{|l|}{ Peak force } \\
\hline Absolute value $\left(10^{3} \mathrm{~N}\right)$ & $1.51 \pm 0.20$ & $1.55 \pm 0.18$ & $1.52 \pm 0.20$ & $1.51 \pm 0.17$ & $2.24 \pm 0.23$ & $1.86 \pm 0.23 * * *$ \\
\hline As a multiple of body weight & $2.13 \pm 0.19$ & $2.23 \pm 0.15^{*}$ & $2.14 \pm 0.21$ & $2.18 \pm 0.15$ & $3.17 \pm 0.30$ & $2.69 \pm 0.30 * * *$ \\
\hline Peak velocity $\left(\mathrm{m} \mathrm{s}^{-1}\right)$ & $2.53 \pm 0.22$ & $2.38 \pm 0.24 * * *$ & $2.31 \pm 0.19$ & $2.20 \pm 0.21 * * *$ & - & \\
\hline \multicolumn{7}{|l|}{ Peak power } \\
\hline Absolute value $\left(10^{3} \mathrm{~W}\right)$ & $3.20 \pm 0.46$ & $3.03 \pm 0.47 * *$ & $2.89 \pm 0.47$ & $2.74 \pm 0.41 *$ & - & \\
\hline Per kg body mass $\left(\mathrm{W} \mathrm{kg}^{-1}\right)$ & $44.4 \pm 6.1$ & $43.0 \pm 6.0$ & $40.0 \pm 5.2$ & $38.9 \pm 5.3$ & - & \\
\hline Jump height (m) & $0.44 \pm 0.07$ & $0.40 \pm 0.08 * *$ & $0.36 \pm 0.06$ & $0.35 \pm 0.08$ & - & \\
\hline
\end{tabular}

Values are mean $\pm \mathrm{SD}$ for 13 men

$* P<0.05, * * P<0.01, * * * P<0.001$ significantly different pre vs. post

force development after the Ironman. The reduced rate of force development might be attributed to glycogen depletion after the Ironman competition (Mueller et al. 2013), whereby glycogen content below a critical level is associated with reductions in sarcoplasmatic reticulum vesicle $\mathrm{Ca}^{2+}$ release rate in endurance athletes (Gejl et al. 2014).

Our finding that CMJ peak velocity decreased despite unaltered CMJ peak force from pre to post Ironman is intriguing and might shed some light on the strategy of the neuromuscular system to cope with the Ironman-induced fatigue. It has been shown that an Ironman decreased leg muscle stiffness due to muscle damage (Garcia-Manso et al. 2011). Conversely, the higher compliance of the muscletendon complex allows a higher storage of elastic energy, which seems to increase jump performance due to a possible increase in efficiency (Bobbert 2001). Therefore, we assume that the recoil of elastic energy was temporarily delayed, leading to a lower peak velocity but a maintained peak force. The observed increase in time interval of $I_{2}$ might support this strategic adaptation: as the rate of force development decreased with fatigue, the neuromuscular system adapts by increasing the impulse interval to reach peak force. Altogether, our results clearly contrast the reasoning of Suzuki et al. (2006), who postulated that the reduced jump height in CMJ and SJ in triathletes after an Ironman event is due to reduced force. The discrepancy in the results might be explained by the different testing protocols. Suzuki et al. (2006) explained their lower jump heights with reduced force measurements during isometric knee extension (open kinetic chain), while no force measurements during the jumping maneuvers were conducted. In contrast, our determination of peak force, peak velocity, and peak power during the jumping maneuver offers the option to explain alterations in jump height with determined variables during the specific task (closed kinetic chain).

Interestingly, the decreases in peak velocity and peak power were similar for the two types of power production, i.e., 'real' power (force production during shortening of the muscle-tendon unit, SJ) and 'reactive' power (force production through a stretch-shortening cycle, CMJ). We therefore deduce that swimming and cycling, which come along with 'real' power production, as well as running ('reactive' power) specifically affected SJ and CMJ performance, respectively, but that there was no difference between SJ and CMJ in terms of performance decrement. Nevertheless, jump height was only reduced during CMJ, which might be indicative of an altered countermovement jumping strategy (e.g., countermovement magnitude). Based on this mechanism, the discrepancy in jump height between CMJ and SJ can be explained by the observed difference in alterations in minimal height between the two jumping maneuvers. Due to muscular fatigue, minimal height during CMJ (i.e., countermovement magnitude) was reduced, which was also shown following fatiguing exercise of the knee extensors (Rodacki et al. 2001). The reduction in minimal height during CMJ in our participants was significantly correlated with the decrease in maximal velocity, which was the main predictor for maximal jump height during CMJ pre and post Ironman. Our result is partially supported by a study of Salles et al. (2011) showing that a lower countermovement magnitude is associated with a lower jump height in the non-fatigued state. In contrast, our participants initiated the SJ from the same starting position and reached the same maximal jump height. Our result of reduced CMJ height after an Ironman is supported by a study from Coso et al. (2012), which reported that maximal jump height during CMJ is already reduced after a half-distance Ironman triathlon.

Absolute peak force during CMJ was not different pre to post Ironman but peak force as a multiple of body weight increased significantly. This increase in relative peak force can be explained by the significant decrease in body mass during the Ironman. As described in a previous study (Mueller et al. 2013), the loss of body mass after an 
Fig. 3 Relationship between peak velocity and jump height during countermovement jumping $\mathbf{a}$ pre and $\mathbf{b}$ post Ironman
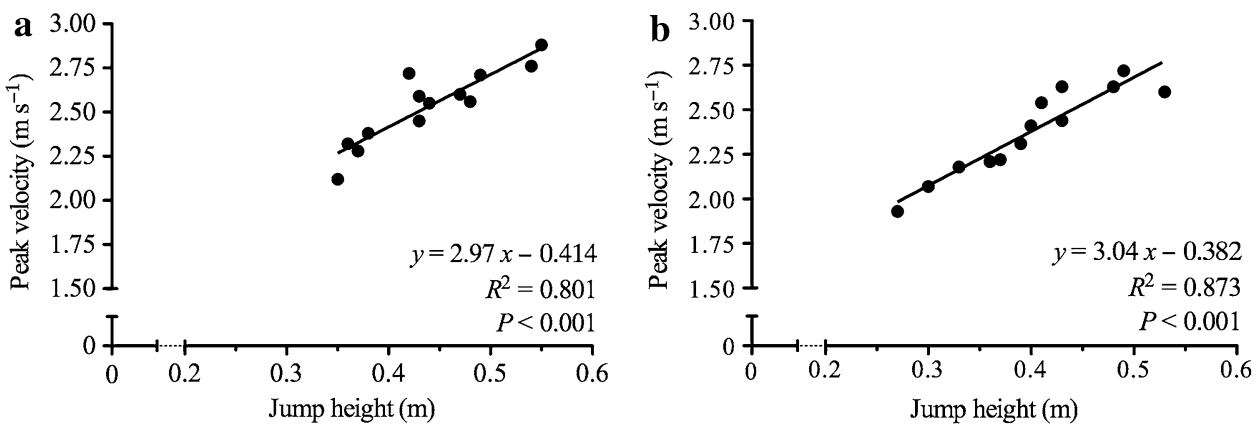

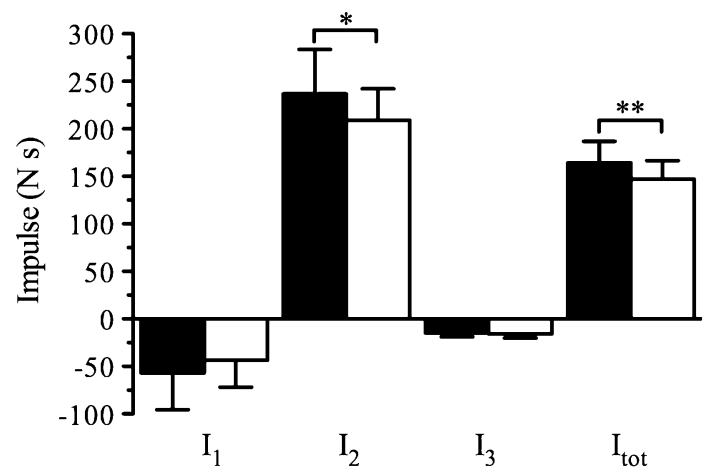

Fig. 4 Pre (black bars) and post (white bars) Ironman results for the three single $\left(I_{1}, I_{2}, I_{3}\right)$ and the total impulse $\left(I_{\mathrm{tot}}\right)$

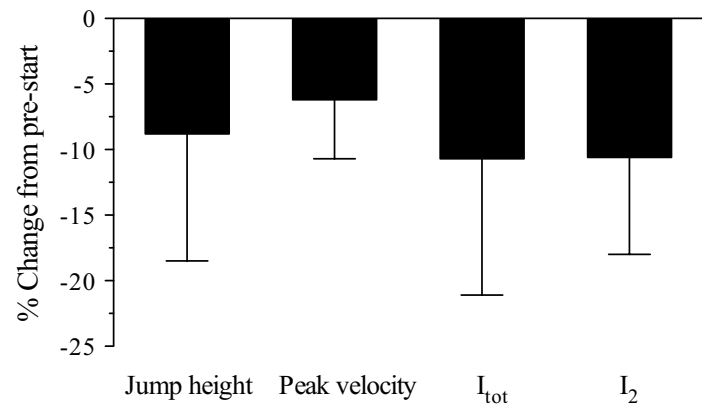

Fig. 5 Relative changes pre to post Ironman in countermovement jump (CMJ) height, peak velocity during CMJ, total impulse $\left(I_{\text {tot }}\right)$, and positive impulse $\left(I_{2}\right)$ during $\mathrm{CMJ}$

Ironman race is attributed to decreases in fat mass, total body water, and glycogen, while muscle protein is not lost during the race. Theoretically, an identical number of contractile units remained present that might be used for force production. The maintenance of absolute peak force, which was notably similar during $\mathrm{CMJ}$ and $\mathrm{SJ}$, points to a specific mechanism of force control during these jumping maneuvers as response to fatigue. This specific mechanism seems to overcome the fatigue-induced influence of several factors (e.g., oxidative stress and inflammation), which are

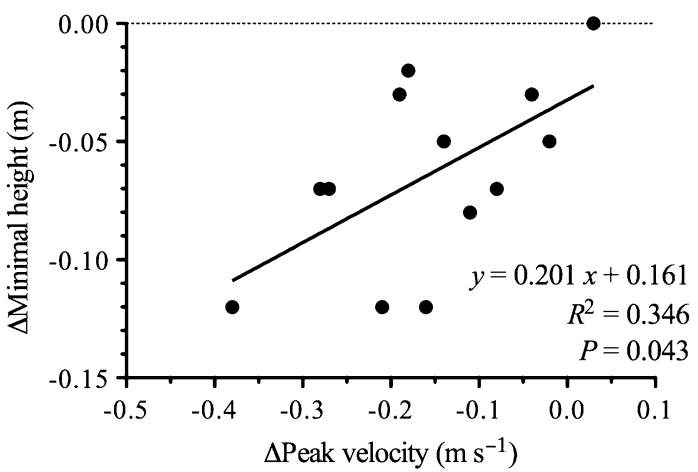

Fig. 6 Relationship between the change in minimal height and the change in peak velocity in an Ironman competition

increased after an Ironman competition (Pinho et al. 2010), on peak force. In relation to peak force during $\mathrm{m} 1 \mathrm{LH}$, peak force during the two-legged jumping maneuvers is of submaximal nature and there is theoretically the possibility to increase this force. To compensate for the fatigue-induced decrease in peak velocity, it would be reasonable if peak force would be increased to achieve peak power. In turn, it appears that peak force during two-legged jumping has a certain set point, which might be controlled by a motor control system. This mechanism might aim at preventing injuries of the musculoskeletal system during fatigue.

While we did not find any change in peak jump force for CMJ and SJ, maximum force during the m1LH maneuvers decreased significantly. As m1LH yields the highest voluntary jumping force acting on the forefoot, it is used to estimate maximal voluntary ground reaction force (Anliker et al. 2011; Anliker and Toigo 2012). The measured decrease in stiffness during m1LH might provide further information about the underlying fatigue mechanisms because leg stiffness is negatively correlated with contact time and positively correlated with maximal ground reaction force (Rabita et al. 2011). Based on a theoretical model (Morgan 1990) and animal experiments (Gregory et al. 2007), we assume that the reduced stiffness was due to muscle damage (i.e., overstretching or disruption of intracellular muscle structures after exercise), which is a 
well-defined phenomenon after an Ironman triathlon (Neubauer et al. 2008) and which leads to a higher compliance of the muscle-tendon complex. Consequently, we assume that muscle damage was the cause for the decrease in the stiffness of the muscle-tendon complex and accordingly, $F_{\mathrm{m} 1 \mathrm{LH}}$. Our result is in line with previous studies investigating the effect of exhausting stretch-shortening cycle exercise (Avela and Komi 1998; Toumi et al. 2006) or an exhaustive run (Rabita et al. 2011) on leg stiffness.

Clearly, further investigations are needed to quantify the relative contributions of the single mechanisms mentioned above to the reduction of maximum force. In future studies, one very important aspect related to the definitions of "peak" vs. "maximum" should be considered: as our data unequivocally showed, there existed an important difference in the peak force that was generated during the different jumping maneuvers. In fact, m1LH yielded force values (per forefoot) that were approximately three times higher than during CMJ or SJ (Anliker and Toigo 2012). Thus, both CMJ and SJ are submaximal jumps with respect to force. Therefore, these jumping maneuvers should not be (mis-) used to measure the effect of interventions on maximum voluntary force, as long as maximum force does not decrease to approximately $30 \%$ of its initial level. In the present study, this difference was seen in the decrement in maximum voluntary force, which manifested itself only during $\mathrm{m} 1 \mathrm{LH}$ and not during the two-legged jumping maneuvers.

The present study has at least two limitations. First, several physiological characteristics (e.g., $\dot{V} \mathrm{O}_{2 \text { peak }}$ ) could not be determined in these participants because pre-testing took place 2-3 days before the competition and the participants were not willing to perform a test to volitional exhaustion. In addition, it was not possible to characterize training history as only few participants were keeping a detailed and systematic training diary. However, according to race performance and their individual history and experience with Ironman competitions, the athletes can be classified as experienced ultra-endurance athletes. Second, the participants were not allowed to drink and eat before completion of the performance test. Since the participants had to travel from the finish line to the laboratory on an individual basis, we cannot be absolutely sure that no nutrients or fluids were consumed. However, if participants had arrived to the laboratory with partly replenished glycogen stores, the obtained results would rather strengthen than weaken our interpretations.

\section{Conclusions}

An Ironman triathlon resulted in a reduction in CMJ height, CMJ peak velocity, and $F_{\mathrm{m} 1 \mathrm{LH}}$. The reduced CMJ height was a result of the lower positive impulse. Therefore, the neuromuscular deficit after the race was due to impairments in force transmission, resulting in a lower average positive force during CMJ, because of a slower rate of force development. The decreased $F_{\text {mlLH }}$ could be partly explained by reduced leg stiffness.

Conflict of interest The authors declare that they have no conflict of interest.

\section{References}

Anliker E, Toigo M (2012) Functional assessment of the musclebone unit in the lower leg. $\mathrm{J}$ Musculoskelet Neuronal Interact 12(2):46-55

Anliker E, Rawer R, Boutellier U, Toigo M (2011) Maximum ground reaction force in relation to tibial bone mass in children and adults. Med Sci Sports Exerc 43(11):2102-2109

Avela J, Komi PV (1998) Reduced stretch reflex sensitivity and muscle stiffness after stretch-shortening cycle exercise in humans. Eur J Appl Physiol 78(5):403-410

Bobbert MF (2001) Dependence of human squat jump performance on the series elastic compliance of the triceps surae: a simulation study. J Exp Biol 204(3):533-542

Coso JD, Gonzalez-Millan C, Salinero JJ, Abian-Vicen J, Soriano L, Garde S, Perez-Gonzalez B (2012) Muscle damage and its relationship with muscle fatigue during a half-iron triathlon. PLoS One 7(8):e43280

Garcia-Manso JM, Rodriguez-Ruiz D, Rodriguez-Matoso D, de Saa Y, Sarmiento S, Quiroga M (2011) Assessment of muscle fatigue after an ultra-endurance triathlon using tensiomyography (TMG). J Sports Sci 29(6):619-625

Gejl KD, Hvid LG, Frandsen U, Jensen K, Sahlin K, Ørtenblad N (2014) Muscle glycogen content modifies SR Ca ${ }^{2+}$ release rate in elite endurance athletes. Med Sci Sports Exerc 46(3):496-505

Gillum TL, Dumke CL, Ruby BC (2006) Muscle glycogenolysis and resynthesis in response to a half Ironman triathlon: a case study. Int J Sports Physiol Perform 1(4):408-413

Gregory JE, Morgan DL, Allen TJ, Proske U (2007) The shift in muscle's length-tension relation after exercise attributed to increased series compliance. Eur J Appl Physiol 99(4):431-441

Hiller WD, O'Toole ML, Fortess EE, Laird RH, Imbert PC, Sisk TD (1987) Medical and physiological considerations in triathlons. Am J Sports Med 15(2):164-167

Knechtle B, Baumann B, Wirth A, Knechtle P, Rosemann T (2010) Male ironman triathletes lose skeletal muscle mass. Asia Pac J Clin Nutr 19(1):91-97

Margaritis I, Tessier F, Verdera F, Bermon S, Marconnet P (1999) Muscle enzyme release does not predict muscle function impairment after triathlon. J Sports Med Phys Fit 39(2):133-139

Montgomery WH 3rd, Pink M, Perry J (1994) Electromyographic analysis of hip and knee musculature during running. Am J Sports Med 22(2):272-278

Morgan DL (1990) New insights into the behavior of muscle during active lengthening. Biophys J 57(2):209-221

Mueller SM, Anliker E, Knechtle P, Knechtle B, Toigo M (2013) Changes in body composition in triathletes during an Ironman race. Eur J Appl Physiol 114(1):123-133

Neubauer O, König D, Wagner KH (2008) Recovery after an Ironman triathlon: sustained inflammatory responses and muscular stress. Eur J Appl Physiol 104(3):417-426

Pinho RA, Silva LA, Pinho CA, Scheffer DL, Souza CT, Benetti M, Carvalho T, Dal-Pizzol F (2010) Oxidative stress and 
inflammatory parameters after an Ironman race. Clin J Sport Med 20(4):306-3011

Raasch CC, Zajac FE, Ma B, Levine WS (1997) Muscle coordination of maximum-speed pedaling. J Biomech 30(6):595-602

Rabita G, Slawinski J, Girard O, Bignet F, Hausswirth C (2011) Spring-mass behavior during exhaustive run at constant velocity in elite triathletes. Med Sci Sports Exerc 43(4):685-692

Rodacki AL, Fowler NE, Bennett SJ (2001) Multi-segment coordination: fatigue effects. Med Sci Sports Exerc 33(7):1157-1167

Salles AS, Baltzopoulos V, Rittweger J (2011) Differential effects of countermovement magnitude and volitional effort on vertical jumping. Eur J Appl Physiol 111(3):441-448
Suzuki K, Peake J, Nosaka K, Okutsu M, Abbiss CR, Surriano R, Bishop D, Quod MJ, Lee H, Martin DT, Laursen PB (2006) Changes in markers of muscle damage, inflammation and HSP70 after an Ironman triathlon race. Eur J Appl Physiol 98(6):525-534

Toumi H, Poumarat G, Best TM, Martin A, Fairclough J, Benjamin M (2006) Fatigue and muscle-tendon stiffness after stretchshortening cycle and isometric exercise. Appl Physiol Nutr Metab 31(5):565-572 